\title{
Im Notfall bitte ein Männerteam!
}

\section{Bernhard Gurtner}

«Frauen schneiden bei Reanimationsmassnahmen schlechter ab». Die am 8. Mai 2017 etwas unbedacht formulierte Presse-Mitteilung der Universität Basel hat eine Schweizer Tageszeitung sofort zur Schlagzeile «Im Notfall bitte ein Männerteam!» veranlasst und auch international hämische Medienkommentare ausgelöst. Ein Macho-Vorurteil, das angesichts der oft bewundernswerten Einsätze professionell ausgebildeter weiblicher Rettungskräfte (Ambulanzen, REGA, Notfallstationen, IPS- und Anäs-

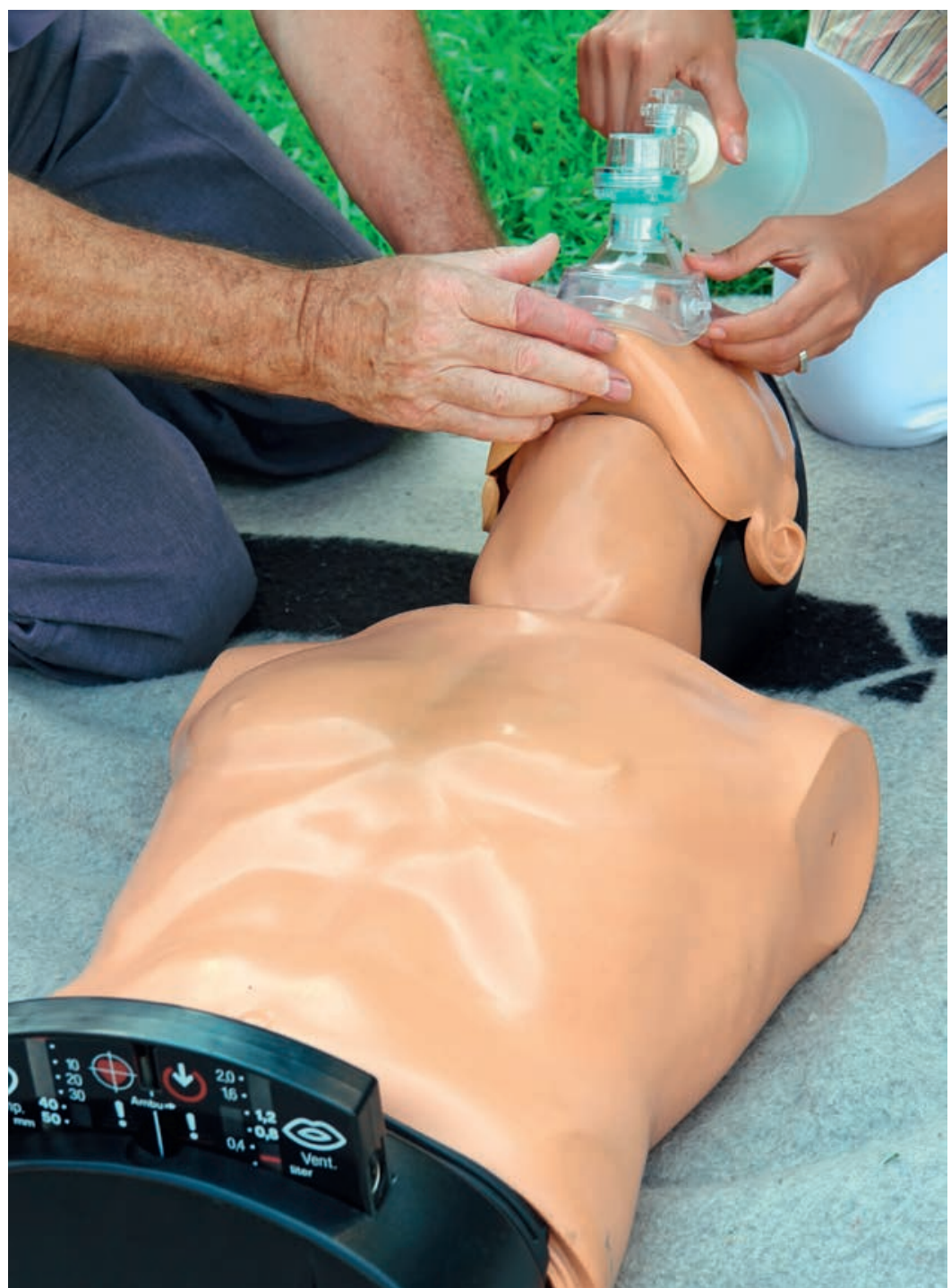

In einer Studie konnte nachgewiesen werden, dass Frauen bei simulierten Reanimationen mehr Schwierigkeiten haben, entschlossen Hand anzulegen und zu führen, als ihre männlichen Kollegen.
thesie-Fachfrauen) negative Einzelerfahrungen unfair verallgemeinert.

Noch so perfekte Simulationen können aber den maximalen Adrenalinausstoss im Ernstfall nie ganz vorwegnehmen.

Die erwähnte, von Frau Prof. Sabina Hunziker geleitete Studie betraf keine echten Notfälle, sondern Reanimationsmassnahmen an Übungspuppen. Zum Einsatz kamen je 108 männliche und weibliche Medizinstudierende, aufgeteilt in 72 Dreiergruppen, teils nur durch Männer oder Frauen gebildet, teils beidgeschlechtlich gemischt. Gemessen wurde die Dauer effektiver Herzdruck-Massage in den ersten drei Minuten nach Alarmauslösung.

\section{Frauen intervenieren weniger resolut}

Es hat sich gezeigt, dass Studentinnen in simulierten Notsituationen weniger beherzt zugreifen und sich scheuen, die Führung spontan zu übernehmen. Ihre Kollegen haben das vielleicht schon bei den Pfadi oder im Militär an Postenläufen gelernt. Könnte es sein, dass Männer solche Übungen am Phantom als sportlichen Wettkampf auffassen, den sie unbedingt gewinnen wollen, indes Frauen weniger Lust haben, ein grosses Plastik-Baby aus fingierter Not zu retten? Es gibt auch - unabhängig vom Geschlecht - skeptische Charakter, die simulierte Situationen und Rollenspiele nie wirklich ernst nehmen können, obwohl solche seit 2011 sogar am Staatsexamen mit «standardisierten Patienten" (SP) vorgeschrieben sind. Wenn eine Schauspielerin grauenhafte Schmerzen allzu dramatisch vortäuscht, dürfen die Kandidaten keinesfalls lachen, weil für eine gute Benotung viel Empathie glaubhaft demonstriert werden muss.

Zweifellos ist es aber unerlässlich, dass im Studium und an späteren Einsatzorten nicht nur technische Fertigkeiten [2], sondern auch Teamwork, Leadership und Kommunikation unter Stress regelmässig geübt werden, ohne dass geplagte Patienten als Versuchskaninchen herhalten müssen.

Stephen Abrahamson, Gründer der Division of Research in Medical Education an der University of Southern California in Los Angeles, hat schon 1966 weltweit als Erster einen computerisierten Patientensimulator 
gebaut. Die menschengrosse Puppe SIM ONE zeigte variable Atembewegungen und Pupillenreaktionen. Herzrhythmus, Blutdruck sowie Karotis- und Temporalispulse liessen sich kritisch verändern. Der Unterkiefer war für Intubationen beweglich. 272000 Dollar kostete der Prototyp, subventioniert vom US Office of Education, weil die Gesundheitsbehörden einen Kredit abgelehnt hatten. Obwohl sich der grosse didaktische Nutzen des Geräts leicht nachweisen liess [1], war das schockierende Phantom für Kollegen und Kommerz eine unerwünschte Frühgeburt. Steve Abrahamson hat dennoch visionär den Fortschritt $\mathrm{zu}$ immer lebensechteren, interaktiven Übungspuppen eingeleitet. Er nutzte aber auch das lokale Angebot an arbeitslosen Hollywood-Schauspielerinnen, um sie als Pseudo-Patientinnen für die Schulung von Anamnese und Status nach vorgegebenem Script sinnvoll zu beschäftigen.

Am Universitätsspital Basel hat der damalige Professor für Anästhesiologie und Reanimation, Daniel Scheidegger - heute Präsident der SAMW -, in Zusammenarbeit mit Swissair und NASA viele bewährte Konzepte des Pilotentrainings und des Umgangs mit Fehlern oder Beinahe-Zwischenfällen in die Medizin übernommen. Ab 1990 wurde in einem simulierten OP die Teamarbeit an Reanimationspuppen nach Checklisten geübt und mit Video erfasst, um Mängel der Kommunikation und der Leadership aufzudecken, die als menschliches Versagen oft gefährlicher sind als technische Fehlleistungen. Als simBa entwickelte sich die Institution in den folgenden Jahren zum anerkannten Schweizerischen Zentrum für medizinische Simulation.

Noch so perfekte Simulationen können aber den maximalen Adrenalinausstoss im Ernstfall nie ganz vor- wegnehmen. Piloten im Flugsimulator wissen, dass sie nach Fehlern virtuell abstürzen, real aber «nur» in der Prüfung durchfallen werden. Medizinische Übungspuppen sterben nicht, wenn das REA-Team verzögert und falsch kooperiert. Dem Chaos bei echten Notfällen

\section{Medizinische Übungspuppen sterben nicht,} wenn das REA-Team verzögert und falsch kooperiert.

kann vorgebeugt werden, wenn Reanimationen überraschend ohne Voranzeige geübt werden.

Die jeweils bestgeeignete Person soll die Leitung ad hoc übernehmen, Aufgaben delegieren und ohne zwingenden Grund nicht selber Hand anlegen, um die Übersicht zu bewahren. Wenn diese Aufgabe bereits von einer routinierten Frau erfüllt wird, besteht kein Anlass, die Teamleitung unterwürfig an einen später hinzukommenden, vielleicht weniger geschulten Assistenten zu übertragen - nur weil er ein Mann ist [3]. Wer aber fällt jeweils den (mannhaften?) Entscheid, ob eine Reanimation begonnen oder abgebrochen wird [4]?

\section{Referenzen}

1 Abrahamson S, Denson JS, Wolf RM. Effectiveness of a simulator in training anaesthesiology residents. Journal of Medical Education. 1969;44(6):515-9.

2 Taverna E, Simulierte Realität. Schweiz Ärztezeitung. 2017;98(18):584-5.

3 Hunziker S, Tschan F, Semmer NK, Marsch S. Importance of leadership in cardiac arrest situations: from simulation to real life and back. Swiss Med Wkly. 2013;143:w13774.

4 Gurtner B. REA ja oder nein? Schweiz Ärztezeitung. 2008;89(34):1473.

Bildnachweis

(C) Roman Milert | Dreamstime.com 